\title{
Two bifurcation transitions of the floating half zone convection in a fat liquid bridge of larger $\mathrm{Pr}$
}

\author{
Z.M. Tang ${ }^{\mathrm{a}, *}$, W.R. Hu ${ }^{\mathrm{a}, \mathrm{b}}, \mathrm{N}$. Imaishi ${ }^{\mathrm{b}}$ \\ a Institute of Mechanics, Chinese Academy of Sciences, Beijing 100080, People's Republic of China \\ ${ }^{\mathrm{b}}$ Institute of Advanced Material Study, Kyushu University, Kasuga, Fukuoka 816-8580, Japan
}

Received 10 February 2000; received in revised form 18 May 2000

\begin{abstract}
The transient process of the thermocapillary convection was obtained for the large $\operatorname{Pr}$ floating half zone by using the method of three-dimensional and unsteady numerical simulation. The convection transits directly from steady and axisymmetric state to oscillatory flow for slender liquid bridge, and transits first from steady and axisymmetric convection to the steady and non-axisymmetric convection, then, secondly to the oscillatory convection for the fatter liquid bridge. This result implies that the volume of liquid bridge is not only a sensitive critical parameter for the onset of oscillation, but also relates to the new mechanism for the onset of instability in the floating half zone convection even in case of large Prandtl number fluid. (c) 2001 Elsevier Science Ltd. All rights reserved.
\end{abstract}

\section{Introduction}

The thermocapillary convection in a floating half zone as shown in Fig. 1 is a typical subject of microgravity science, and has been studied extensively in the last two decades. The volume of liquid bridge is a sensitive critical geometrical parameter for the onset of oscillatory convection in the floating half zone of large Prandtl number, as analyzed by Cao et al. [1], Monti et al. [2], Hu et al. [3], Shevtsova and Legros [4], Tang and $\mathrm{Hu}$ [5]. The marginal curves for onset of oscillatory thermocapillary convection in case of larger Prandtl number divide into two branches relating, respectively, to the slender liquid bridge and fat liquid bridge as show in Fig. 2. There is typically a gap region between two marginal curves, and the gap region associates with larger critical Marangoni number. However, the gap may be disappeared and two curves connect to form a cusp if the geometrical aspect $A$ is small. The microgravity experiments were performed with the drop shaft facility by Yao et al. [6] and Sakurai and Hirata [7]. Recently, similar conclusion was obtained by the linear instability analysis for the case of the microgravity

\footnotetext{
${ }^{*}$ Corresponding author.
}

environment, but the influence of liquid bridge volume on the onset of oscillation is quite different in cases of smaller Prandtl number [8] in comparison with the cases of large Prandtl numbers [9].

There is usually one bifurcation transition of thermocapillary convection in a liquid bridge of large Prandtl number, that is, the transition from the steady and axi-symmetric thermocapillary convection to the oscillatory convection. The conclusion have been proved by many experiments, at first by Chun and Wuest [10], and also, by Schwabe and Scharmann [11]. The linear instability analysis have been given by Neitzel et al. [12], Wanschura et al. [13], Chen et al. [14], Chen and $\mathrm{Hu}$ [9] and Chen et al. [8]. The energy stability analysis was given by Neitzel et al. [15]. The unsteady and threedimensional numerical simulation were reported by Savino and Monti [16], Yasuhiro et al. [17] and Tang and $\mathrm{Hu}$ [5]. Both experimental and theoretical works were conducted in case of large Prandtl number to study the onset from the steady and axi-symmetric convection to oscillatory convection. Some results support the idea of hydrothermal wave instability, which was suggested early by Smith and Davis [18].

Two bifurcation transitions of thermocapillary convection in a floating half zone of smaller Prandtl number $P r=0.01$ was obtained by the numerical simulation of 


\begin{tabular}{|llll}
\hline Nomenclature & $w$ & dimensional axial velocity \\
$A$ & geometrical aspect ratio & $W$ & dimensionless axial velocity \\
$d_{0}$ & diameter of upper rod and lower rod & $z$ & dimensional axial coordinate \\
$g$ & gravitational acceleration & Greek & symbols \\
$G r$ & Grashof number & $\alpha_{\mathrm{T}}$ & dimensionless heating rate \\
$k$ & thermal diffusion coefficient & $\beta$ & thermal expansion coefficient \\
$l$ & height of liquid bridge & $\delta_{\mathrm{V}}$ & non-axisymmetric degree of velocity \\
$M a$ & Marangoni number & $\delta_{\mathrm{T}}$ & non-axisymmetric degree of temperature \\
$p$ & pressure & $\Delta T$ & temperature difference between upper rod and \\
$P$ & dimensionless pressure & & lower rod \\
$P r$ & Prandtl number & $\Delta T_{\mathrm{c} 1}$ & first critical temperature difference \\
$R e$ & Reynolds number & $\Delta T_{\mathrm{c} 2}$ & second critical temperature difference \\
$r$ & dimensional radial coordinate & $\zeta$ & dimensionless axial coordinate \\
$t$ & dimensional time & $\eta$ & dimensionless azimuthal coordinate \\
$T$ & dimensional temperature & $\theta$ & dimensional azimuthal coordinate \\
$T_{*}$ & dimensional reference temperature & $\Theta$ & dimensionless temperature \\
$T_{0}$ & dimensional temperature of lower rod & $v$ & viscosity coefficient \\
$u$ & dimensional radial velocity & $\xi$ & dimensionless radial coordinate \\
$U$ & dimensionless radial velocity & $\rho$ & density of fluid \\
$v$ & dimensional azimuthal velocity & $\sigma$ & surface tension coefficient \\
$V$ & dimensionless azimuthal velocity & $\tau$ & dimensionless time \\
$v_{*}$ & typical velocity & $\psi$ & stream function \\
$V_{1}$ & volume of liquid bridge & $\Omega$ & vorticity \\
$V_{0}$ & volume of cylindrical liquid bridge & & \\
& & & \\
\hline
\end{tabular}

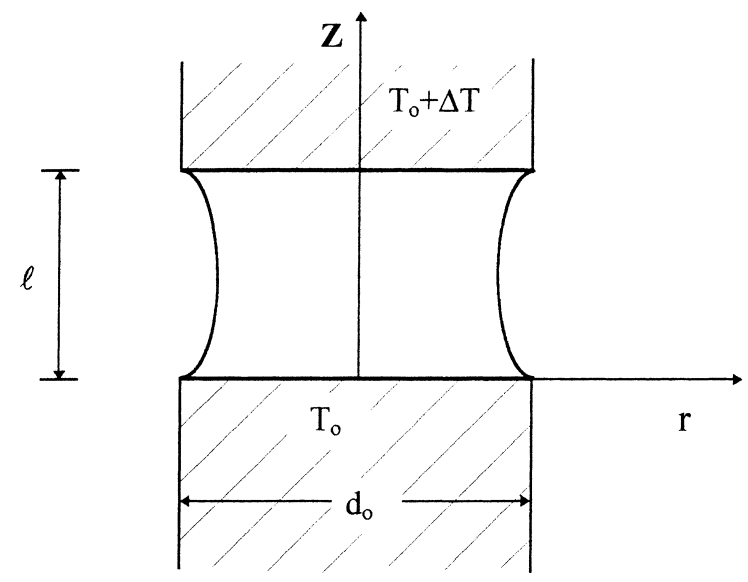

Fig. 1. Schematic diagram of a floating half zone.

Levenstam and Amberg [19], that is, the steady and axisymmetrical convection transits to the steady and asymmetric convection, and then, to the oscillatory convection. The result implies that the bifurcation mechanism in case of small Prandtl number is hydrodynamic instability, but not the hydrothermal instability.

Two bifurcation transitions in a fat liquid bridge of 10 cst silicon oil of larger Prandtl number $\operatorname{Pr}=105.6$ were observed through the numerical simulation in case

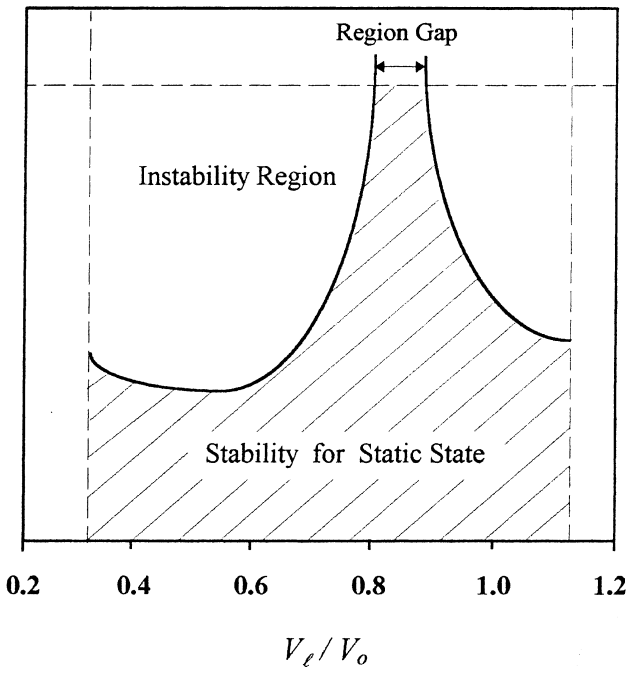

Fig. 2. Typical critical applied temperature difference depending on the volume of a liquid bridge in the floating half zone convection.

of the Earth's gravity condition by Tang and $\mathrm{Hu}$ [20]. The linear instability analysis for a fat liquid bridge gives a steady instability mode of $m=1$ and $\omega_{i}=0$ [21] and this instability mode associates with the first transition from the steady and axisymmetric convection to the 
steady and axial asymmetric convection. These conclusions for larger Prandtl number fluid are similar to the ones for small Prandtl number fluid discussed by Levenstam and Amberg [19]. In the present paper, the unsteady and three-dimensional numerical simulation is applied to discuss the same subject in case of the microgravity environment, and the two bifurcation transitions are obtained. The physical model and the mathematical description are discussed in Section 2. Two bifurcation transitions in a fat liquid bridge of larger Prandtl number are obtained during a fixed heating rate, and the results are discussed in Section 3. The first bifurcation in a fat liquid bridge and the transitions of the liquid bridges with different volume ratios are given, respectively, in Sections 4 and 5. The conclusion and discussion are summarized in Section 6.

\section{Physical model and mathematical description}

A liquid bridge of floating half zone between two coaxis $z$ copper rods of same diameter $d_{0}$ as shown in Fig. 1 is discussed in the present paper, and the liquid bridge has a height $l$. There are two typical geometrical parameter: the geometrical aspect ratio $A=l / d_{0}$ and volume ratio $V=V_{1} / V_{0}$, where $V_{1}$ and $V_{0}$ are, respectively, the volume of liquid bridge and the volume of a cylindrical liquid bridge of $l$ in height and $d_{0}$ in diameter. The lower rod keeps a constant temperature $T_{0}$, and the temperature at the upper rod is $T_{0}+\Delta T$, where positive temperature difference $\Delta T$ may be a constant or change with time. The isothermal case relates to $\Delta T=0$, and the thermocapillary convection is driven by the gradient of surface tension if there is a positive applied temperature difference $\Delta T$, because of the surface tension

$\sigma=\sigma_{0}+(\mathrm{d} \sigma / \mathrm{d} T)\left(T-T_{*}\right)$,

where $T_{*}$ is a constant reference temperature, and $\mathrm{d} \sigma / \mathrm{d} T$ is usually negative. The deviation from the steady and axisymmetric convection may be excited during the increasing of the applied temperature difference $\Delta T$.

The thermocapillary convection in the liquid bridge is controlled by the relationships of mass conservation, momentum conservation and energy conservation. Based on the Boussinesq approximation, the governing equations in the microgravity environment may be written mathematically as follows:

$\nabla \cdot \boldsymbol{v}=0$

$\partial \boldsymbol{v} / \partial t+\boldsymbol{v} \cdot \nabla \boldsymbol{v}=-\nabla(p / \rho)+v \Delta \boldsymbol{v}$,

$\partial T / \partial t+\boldsymbol{v} \cdot \nabla T=\kappa \Delta T$

where $\rho, p, T$ are, respectively, the density, pressure, temperature of the liquid, $\boldsymbol{v}=(u, v, w)$ the velocity vec- tor, $\Delta$ the Laplace operator, and $v$ and $k$ are, respectively, the kinematics viscosity and thermal diffusion coefficients. Eqs. (2.2)-(2.4) may be written in a cylindrical coordinate system as adopted in Fig. 1.

Non-dimensional quantities and parameters are introduced as follows.

$\xi=r / l, \quad \eta=\theta / l, \quad \zeta=z / l, \quad \tau=t /\left(l / v_{*}\right)$,

$U=u / v_{*}, \quad V=v / v_{*}, \quad W=w / v_{*}$,

$P=p / \rho v_{*}^{2}, \quad \Theta=T / \Delta T_{*}$,

$R e^{*}=v_{*} l / v, \quad M a^{*}=v_{*} l / \kappa, \quad \operatorname{Pr}=v / \kappa$,

where the typical velocity $v_{*}$ is defined by the thermocapillary effect as $v_{*}=|\mathrm{d} \sigma / \mathrm{d} T| \Delta T_{*} /(\rho v), \mathrm{d} \sigma / \mathrm{d} T$ a constant, $\Delta T_{*}=T_{*}-T_{0}$ a constant applied temperature difference and $T_{*}$ is a reference constant temperature which is defined as the highest temperature at the upper rod during a heating process. The non-dimensional parameters are related by $M a^{*}=R e^{*} P r$. It is noted that the parameters $R e^{*}$ and $M a^{*}$ are defined by a constant typical velocity $v_{*}$ with a fixed temperature difference $\Delta T_{*}$. The local values for a fixed applied temperature difference $\Delta T$ will be useful, as

$R e=R e^{*} \Delta T / \Delta T_{*}, \quad M a=M a^{*} \Delta T / \Delta T_{*}$.

Introduce the non-dimensional vector stream function $\boldsymbol{\Psi}=\left(\Psi_{r}, \Psi_{\theta}, \Psi_{z}\right)$ and the vector vorticity $\boldsymbol{\Omega}=$ $\left(\Omega_{r}, \Omega_{\theta}, \Omega_{z}\right)$, defined, respectively, as

$\nabla \times \boldsymbol{\Psi}=\boldsymbol{V}, \quad \nabla \times \boldsymbol{V}=\boldsymbol{\Omega}$.

Then, the non-dimensional equations can be written as

$\nabla \times \nabla \times \boldsymbol{\Psi}=\boldsymbol{\Omega}$,

$\partial \boldsymbol{\Omega} / \partial \tau+(\nabla \times \boldsymbol{\Psi}) \cdot \nabla \boldsymbol{\Omega}=\Delta \boldsymbol{\Omega} / R e^{*}$,

$\partial \Theta / \partial \tau+(\nabla \times \boldsymbol{\Psi}) \cdot \nabla \Theta=\Delta \Theta / M a^{*}$.

The boundary conditions are as follows:

$\zeta=0$ and 1 :

$\Psi_{r}=0, \quad \Psi_{\theta}=0, \quad \partial \Psi_{z} / \partial \zeta=0$,

$\Omega_{r}=-\frac{\partial}{\partial \zeta}\left(\frac{\partial \Psi_{r}}{\partial \zeta}-\frac{\partial \Psi_{z}}{\partial \xi}\right)$,

$\Omega_{\theta}=\frac{\partial}{\partial \zeta}\left(\frac{\partial \Psi_{\theta}}{\partial \xi}-\frac{1}{\xi} \frac{\partial \Psi_{z}}{\partial \eta}\right), \quad \Omega_{z}=0$,

$\Theta(\tau, \xi, \eta, 0)=0, \quad \Theta(\tau, \xi, \eta, 1)=f(\tau)$,

$\xi=R(\zeta)$

$\Psi_{s}=0, \quad \Psi_{\theta}=0, \quad \nabla \cdot \Psi=0$, 


$$
\begin{aligned}
\Omega_{r}= & \frac{1}{\xi} \frac{\partial}{\partial \eta}\left(\frac{1}{\xi} \frac{\partial \xi \Psi_{\theta}}{\partial \xi}-\frac{1}{\xi} \frac{\partial \Psi_{r}}{\partial \eta}\right)-\frac{\partial}{\partial \zeta}\left(\frac{\partial \Psi_{r}}{\partial \zeta}-\frac{\partial \Psi_{z}}{\partial \xi}\right), \\
\Omega_{\theta}= & -\left\{\frac{\left(1+R^{2}\right)}{\left(1-R^{2}\right)} \frac{\partial T}{\partial S}+\frac{2 R}{\left(1-R^{2}\right)}\left(\frac{\partial}{\partial \xi}\left(\frac{1}{\xi} \frac{\partial \Psi_{z}}{\partial \eta}-\frac{\partial \Psi_{\theta}}{\partial \zeta}\right)\right.\right. \\
& \left.\left.-\frac{\partial}{\partial \zeta}\left(\frac{1}{\xi} \frac{\partial \xi \Psi_{\theta}}{\partial \xi}-\frac{1}{\xi} \frac{\partial \Psi_{r}}{\partial \eta}\right)+2 \frac{\partial}{\partial \zeta}\left(\frac{\partial \Psi_{z}}{\partial \eta}-\frac{\partial \Psi_{\theta}}{\partial \zeta}\right)\right)\right\} \\
\Omega_{z}= & \frac{\left(1+R^{2}\right)^{1 / 2}}{R} \frac{\partial T}{\partial \eta}+2 \frac{\partial}{\partial \xi}\left(\frac{\partial \Psi_{r}}{\partial \zeta}-\frac{\partial \Psi_{z}}{\partial \xi}\right) \\
& -R\left[\Omega_{r}+2 \frac{\partial}{\partial \zeta}\left(\frac{\partial \Psi_{r}}{\partial \zeta}-\frac{\partial \Psi_{z}}{\partial \xi}\right)\right],
\end{aligned}
$$

$\partial \Theta / \partial n=0$

where the free surface is described as $\xi=r / l=R(\zeta)$ and $R(0)=R(1)=1 /(2 A), \boldsymbol{n}$ and $\boldsymbol{s}$ are, respectively, the unit vector in the normal direction of the free surface and in the direction perpendicular to both $\boldsymbol{n}$ and azimuthal direction of the free surface, and the heating curve in the present paper is given by

$f(\tau)= \begin{cases}\alpha_{\mathrm{T}} \tau, & \tau \leqslant T_{*} / \alpha_{\mathrm{T}} \Delta T_{*}, \\ T_{*} / \Delta T_{*}, & \tau \geqslant T_{*} / \alpha_{\mathrm{T}} \Delta T_{*},\end{cases}$

where the temperature $T_{*}$ and the heating rate $\alpha_{T}$ are constants. According to the definition, the ratio volume of liquid bridge may be written as

$V=4 A^{2} \int R^{2}(\zeta) \mathrm{d} \zeta$.

Then, the problem described by Eqs. (2.8)-(2.10) with boundary conditions (2.11)-(2.16) could be solved for the case of given geometrical parameters $A$ and $V$. The initial condition relates to a static case of isothermal liquid bridge, where the applied temperature difference is zero. Boundary conditions (2.13) and (2.17) consist with the initial condition. The usual case of a cylindrical liquid bridge relates to the condition $R(\zeta)=1 /(2 A)=$ constant, and then $V=1$.

\section{Transient process in a fat liquid bridge}

Based on the linear instability analysis of Chen and $\mathrm{Hu}$ [21], the bifurcation feature is sensitively depended on the volume ratio $V_{1} / V_{0}$, and a steady and axial asymmetric instability mode $m=1$ and $\omega_{i}=0$ is obtained for case of $\operatorname{Pr}=100, V_{1} / V_{0}=1.2$ and $A=0.6$. This result shows that, the instability in this case is associated with the transition from the steady and axisymmetric thermocapillary convection to a steady and asymmetric convection. The linear instability analysis can only give the first instability deviated from the basic state, and the second bifurcation for the onset of oscil- lation can only be performed by the numerical simulation of unsteady and three-dimensional model.

The problem is solved numerically by the finite element method (FEM), with the cell numbers in the $r, \theta$ and $z$ directions are, respectively, 12,16 and 12, and hence, the floating half zone is divided into 10758 tetrahedron elements associated with 2064 nodes. The nonlinear convective terms in the vorticity equation and energy equation are calculated by the characteristic line method, and the diffusion terms are calculated by the FEM.

The thermocapillary convection in a liquid bridge of $12 \mathrm{~mm}$ in height and $15 \mathrm{~mm}$ in diameter is discussed in case of microgravity environment. The dimensional heating rate and reference temperature are adopted, respectively, as $0.05^{\circ} \mathrm{C} / \mathrm{s}$ and $\Delta T_{*}=25^{\circ} \mathrm{C}$. The thermocapillary convection will be driven from the static state of zero temperature difference $\Delta T=0$ at the beginning to the oscillatory state related to the larger temperature difference $\Delta T>(\Delta T)_{\mathrm{c}}$. Typical evolutionary process of temperature and velocity in a slender liquid bridge, for example $V_{1} / V_{0}=0.8$, during the increasing of the applied temperature difference was shown clearly in Fig. 3,
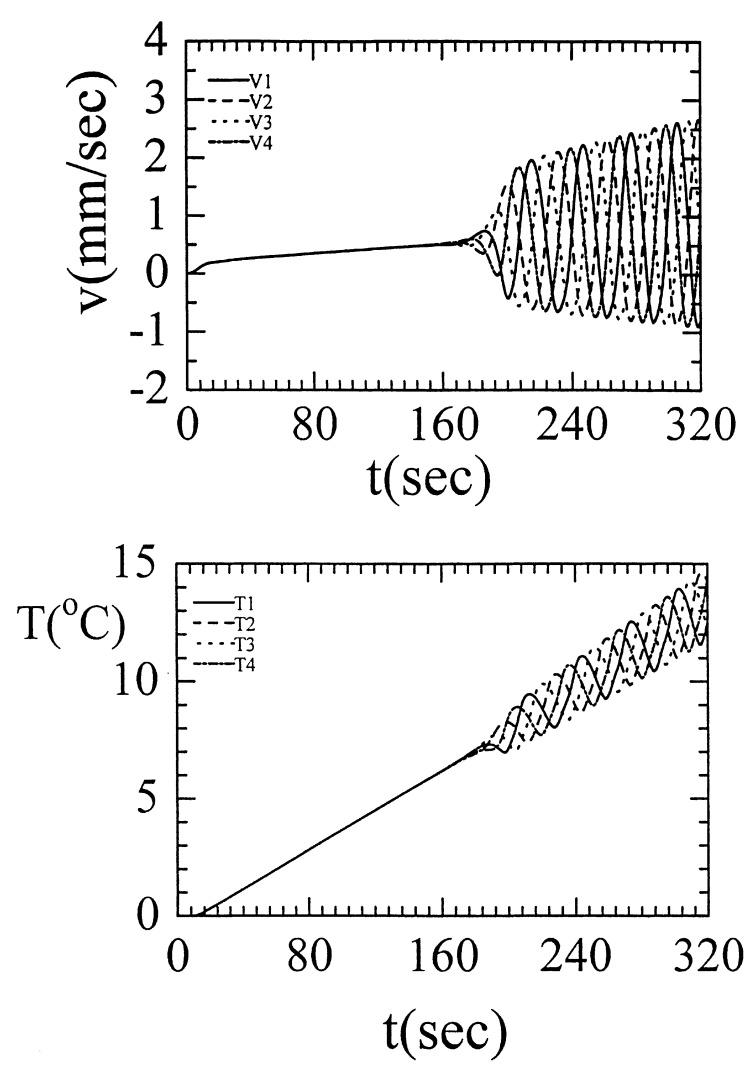

Fig. 3. The onset of oscillatory convection from the steady and axi-symmetric convection in a slender liquid bridge of floating half zone $\left(l / d_{0}=0.8, V_{1} / V_{0}=1.025\right)$. 
where the temperature evolutions are given at four points $\theta=0, \pi / 2, \pi$ and $3 \pi / 2$ on the free surface of the liquid bridge in a cross-section $\zeta=0.55$. Four curves of surface temperatures coincide before the onset of oscillation, and then separate to a phase difference of $\pi / 2$ one by another. The results show clearly the onset of oscillatory convection from the steady and symmetric state to oscillatory convection, and there is only one bifurcation of transition in the slender liquid bridge.

Similar analysis is applied to the case of a fat liquid bridge $V_{1} / V_{0}=1.025$, which relates to the right curve of Fig. 9. The evolutionary processes of the azimuthal velocity components and the temperatures on the free surface in a cross-section $\zeta=0.55$ are shown in the upper and lower of Fig. 4. Four evolutionary profiles at the boundary of a cross-section are not overlapped together before the onset of oscillation, and change slowly with time. The results of Fig. 4 mean that two bifurcation transitions appear during the increasing of applied temperature difference, and there is a period related to the quasi-steady and axial asymmetric convection before the onset of oscillation.
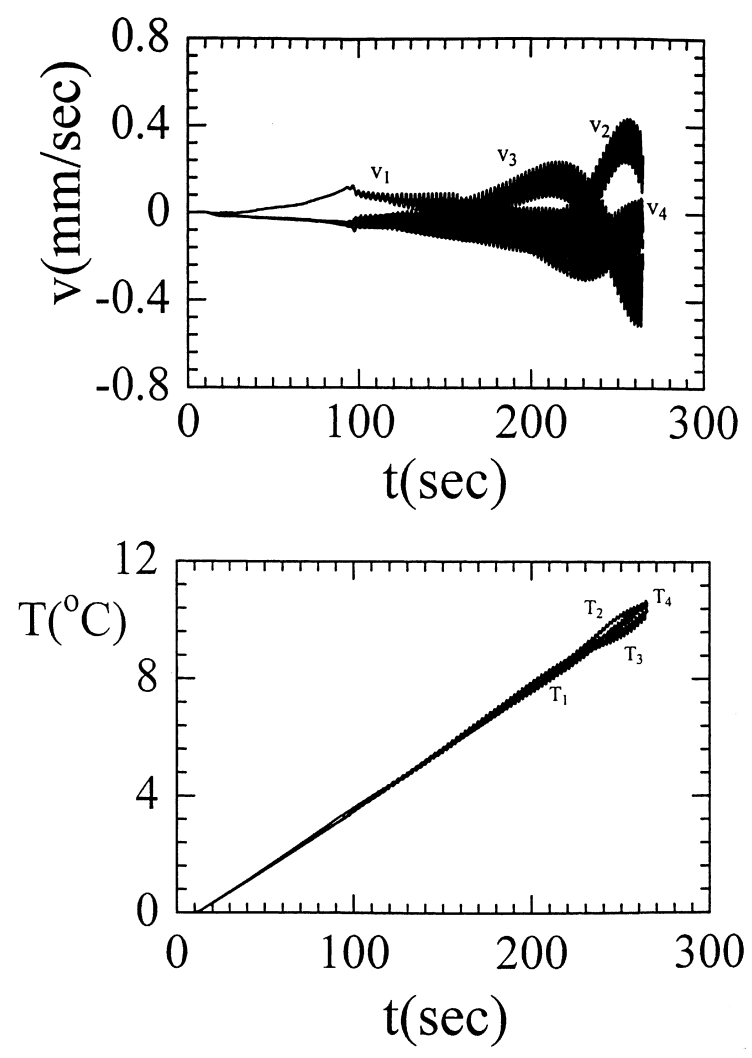

Fig. 4. The transient feature of two bifurcation given by the temperature (upper) and azimuthal velocity (lower) in a fat liquid bridge of floating half zone $\left(l / d_{0}=0.8, V_{1} / V_{0}=1.025\right)$.
For the quantitative description of the first bifurcation, the non-axisymmetric degrees are introduced, respectively, for azimuthal velocity and temperature as follows:

$\delta_{\mathrm{V}}=\frac{V_{\max }-V_{\min }}{V^{*}}, \quad \Delta_{\mathrm{T}}=\frac{T_{\max }-T_{\min }}{\Delta T}$,

where subscript max and min denote, respectively, the maximum and minimum values on the free surface of a cross-section $\zeta=0.55, \mathrm{~V}$ and $\mathrm{T}$ are, respectively, the azimuthal component of velocity and temperature, and $V^{*}$ is the maximum velocity in the liquid bridge at a certain applied temperature difference $\Delta t$. Both $\delta_{\mathrm{T}}$ and $\delta_{\mathrm{v}}$ are zero in the axisymmetric convection, and increase gradually during the onset of the first bifurcation, which relates to the transition from the steady and symmetric convection to the quasi-steady and axial asymmetric convection. Evolution of the non-symmetric degree $\delta_{\mathrm{V}}$ and $\delta_{\mathrm{T}}$ are given in Fig. 5, which shows clearly the two processes of the onset of steady and axial asymmetric states and the onset of oscillatory states. The first critical applied temperature difference $\delta_{\mathrm{T}_{\mathrm{cl}}}$ may be defined by the moment when $\delta_{\mathrm{T}}$ equals 0.02 , and the critical value is

$\Delta T_{\mathrm{c} 1}=0.773^{\circ} \mathrm{C}$.
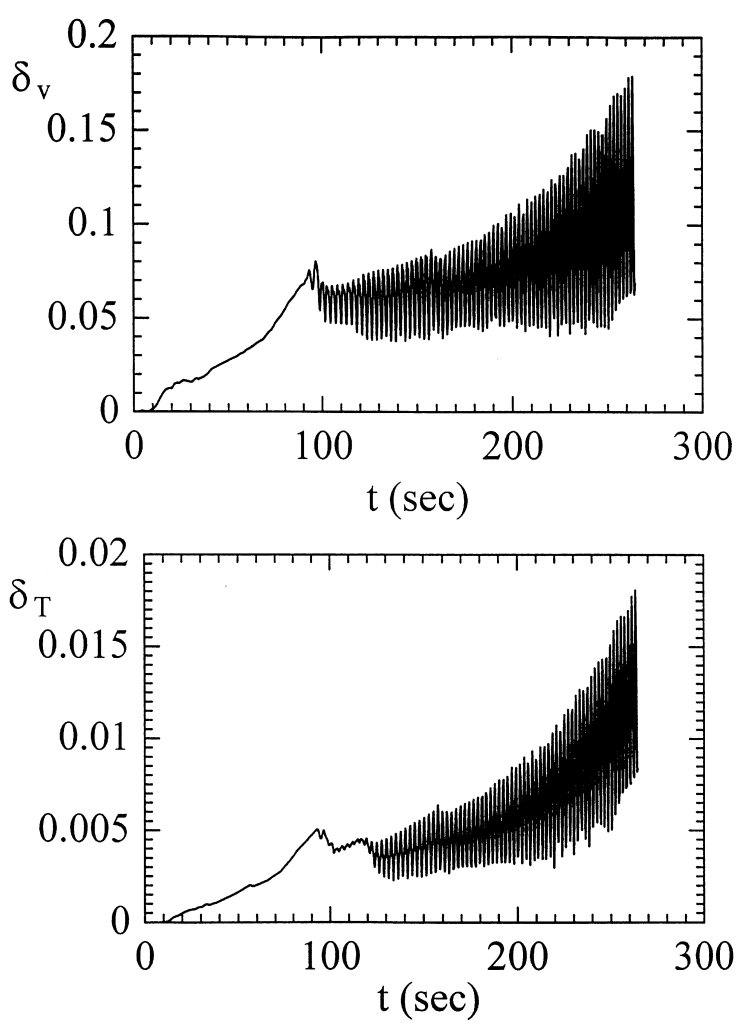

Fig. 5. The transient process described by the parameters $\delta_{\mathrm{V}}$ and $\delta_{\mathrm{T}}$ in a fat liquid bridge $\left(l / d_{0}=0.8, V_{1} / V_{0}=1.025\right)$. 
The second bifurcation for the onset of oscillatory convection from the quasi-steady and axial asymmetric convection may be given as usually by the oscillatory amplitude, and it gives

$\Delta T_{\mathrm{c} 2}=4.78^{\circ} \mathrm{C}$.

Two critical values of the applied temperature difference associate with two corresponding critical Marangoni values, i.e.

$M a_{\mathrm{c} 1}=628.67, \quad M a_{\mathrm{c} 2}=3886.68$.

The results give two critical transients of thermocapillary convection in a fat liquid bridge of larger Prandtl number, similar to the one in a cylindrical liquid bridge of small Prandtl number as given by Levenstam and Amberg [19]. However, the onset of bifurcation in case of larger Prandtl number will be induced obviously by both the hydrodynamic and thermal effects, not only by the hydrodynamic effect as suggested in the case of low Prandtl number. Furthermore, the conclusion on appearance of first bifurcation agrees with the results of linear instability analysis, given by Chen and $\mathrm{Hu}$ [21], that is, the steady and axisymmetric thermocapillary convection may be unstable as composed to a steady and axial asymmetric thermocapillary convection. The conclusion of present paper does not support the mechanism of hydrothermal instability, which requires a traveling wave.

\section{The first bifurcation in a fat liquid bridge}

The states of thermocapillary convection respond to a fixed heating rate of $0.05^{\circ} \mathrm{C} / \mathrm{s}$ is discussed in Section 3, and a quasi-steady and axial asymmetric convection has been obtained. The steady and axial asymmetric convection is beneficial to understanding the first bifurcation, and may be obtained if the applied temperature difference is given specially. Based on the results of Eqs. (3.2) and (3.3), the quasi-steady and axial asymmetric convection is in the temperature range $0.778^{\circ} \mathrm{C}<$ $\Delta T<4.78^{\circ} \mathrm{C}$.

To discuss the first bifurcation in details, a heating process is designed to keep the temperature difference $\Delta T$ falls in the region of steady and axial asymmetric state, and $\Delta T$ is adopted as $\Delta T=3.5^{\circ} \mathrm{C}$ as an example. In this case, the applied temperature difference increases from zero at the beginning to $3.5^{\circ} \mathrm{C}$ at $70 \mathrm{~s}$, and then keeps at the $3.5^{\circ} \mathrm{C}$ afterwards. The evolutionary azimuthal velocities and temperatures at four points $\theta=0$, $\pi / 2, \pi$ and $3 \pi / 2$ on the free surface at cross-section $\zeta=0.55$ are show in Fig. 6, and the steady and axial asymmetric thermocapillary convection is persisted in a long period of only slowly variation, for example over 201 s. This results mean that, the steady and axial
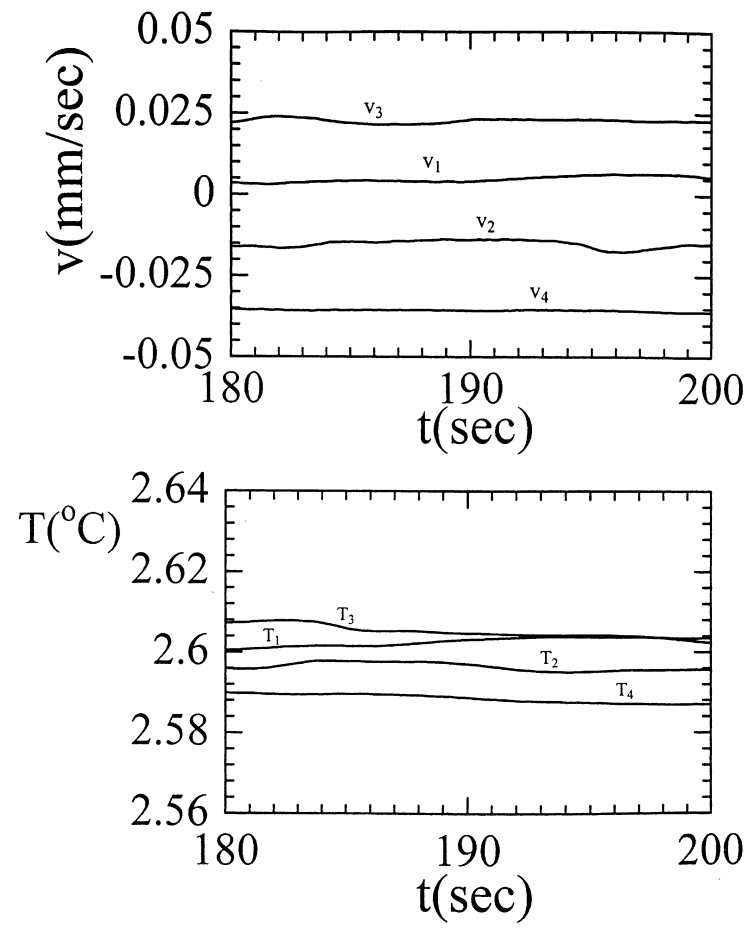

Fig. 6. The evolutions of azimuthal velocity (upper) and temperature (lower) for a fixed temperature difference $\Delta T=3.5^{\circ} \mathrm{C}$ after a heating process with heating rate $=0.05^{\circ} \mathrm{C} / \mathrm{s}$ in a fat liquid bridge $\left(l / d_{0}=0.8, V_{1} / V_{0}=1.025\right)$.

asymmetric convection is a real state, which may be persisted in a long period.

The temperature distributions and the velocity distributions in the fat liquid bridge $V_{1} / V_{0}=1.025$ at $\Delta T=3.5^{\circ} \mathrm{C}$ are given, respectively, in Figs. 7 and 8 at the moment $t=156.9 \mathrm{~s}$ (left figures) and $t=201.4 \mathrm{~s}$ (right figures). The distributions of the steady and axial asymmetric convection keep nearly the same during the process. The steady and axial asymmetric mode in Figs. 7 and 8 relates to $m=1$, and this conclusion agrees with the result of the linear instability analysis given by Chen and $\mathrm{Hu}[21]$.

\section{The transient processes of different volume-ratio liquid bridge}

The critical temperature differences depending on the volume ratios of the liquid bridge with $A=0.8$ are given in Fig. 9. $\Delta T_{\mathrm{cl}}$ is defined as the first critical temperature difference related to the transition from steady and axisymmetric convection to the three-dimensional and steady convection, and $\Delta T_{\mathrm{c} 2}$ is the second critical temperature difference from three-dimensional and steady convection to the three-dimensional, oscillatory con- 

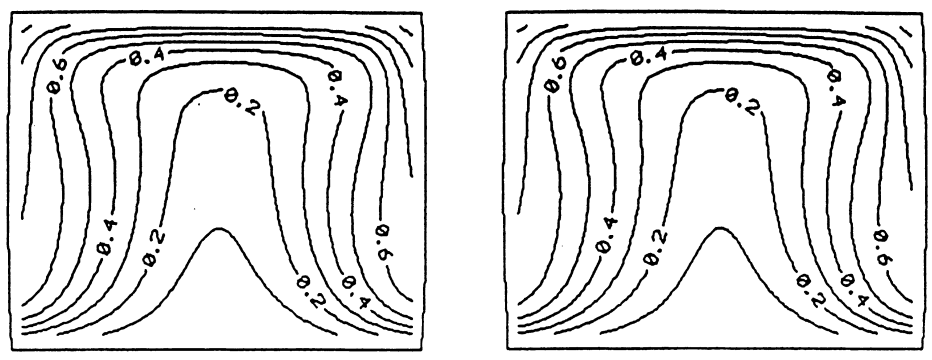

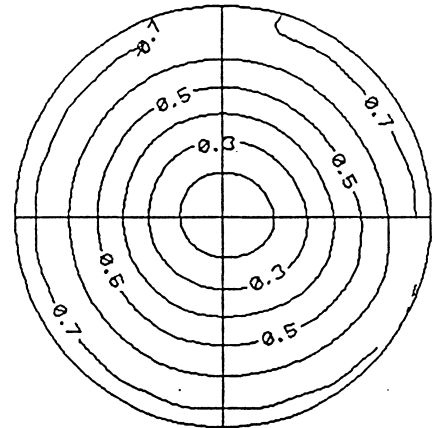

(a) $\mathrm{t}=156.9 \mathrm{sec}$

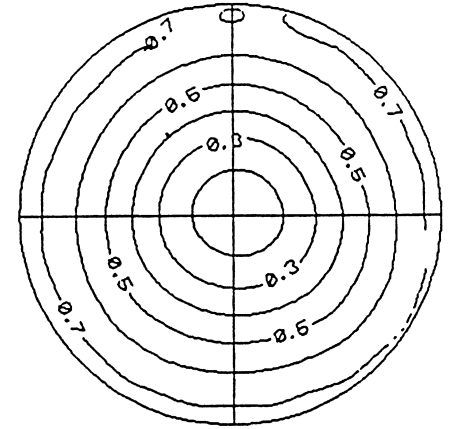

(b) $\mathrm{t}=201.4 \mathrm{sec}$

Fig. 7. The steady and axial asymmetric distribution of temperature in a fat liquid bridge $\left(l / d_{0}=0.8, V_{1} / V_{0}=1.025, \Delta T=3.5^{\circ} \mathrm{C}\right):(\mathrm{a})$ the temperature distribution in the vertical section $0-180^{\circ}$ (upper), and (b) the temperature distribution in the section $z / l=0.55$ (lower).
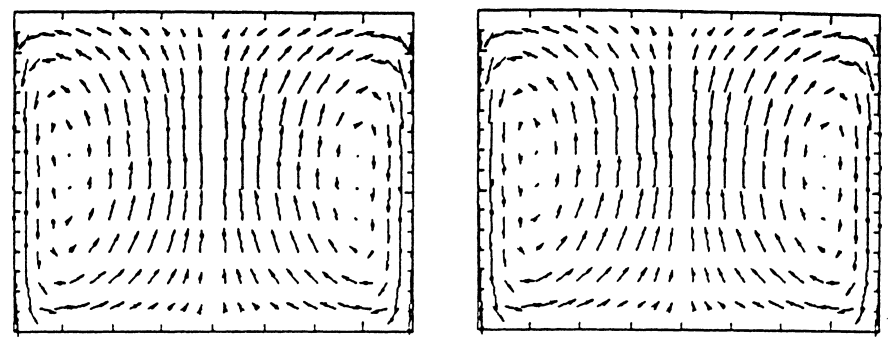

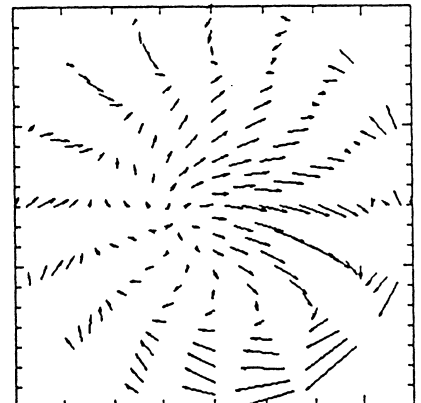

(a) $\mathrm{t}=156.9 \mathrm{sec}$

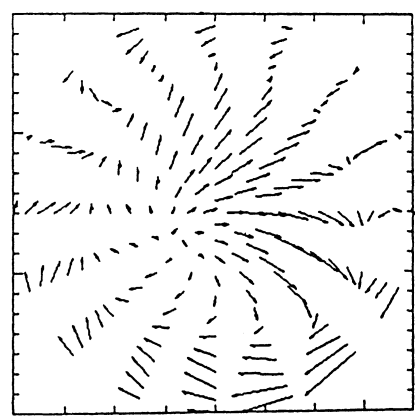

(b) $\mathrm{t}=201.4 \mathrm{sec}$

Fig. 8. The steady and axial asymmetric distribution of velocity in a fat liquid bridge $\left(l / d_{0}=0.8, V_{1} / V_{0}=1.025, \Delta T=3.5^{\circ} \mathrm{C}\right):(\mathrm{a})$ the flow field in the vertical section $0-180^{\circ}$ (upper), and (b) the distribution of azimuthal velocity (lower) in the section $z / l=0.55$. 


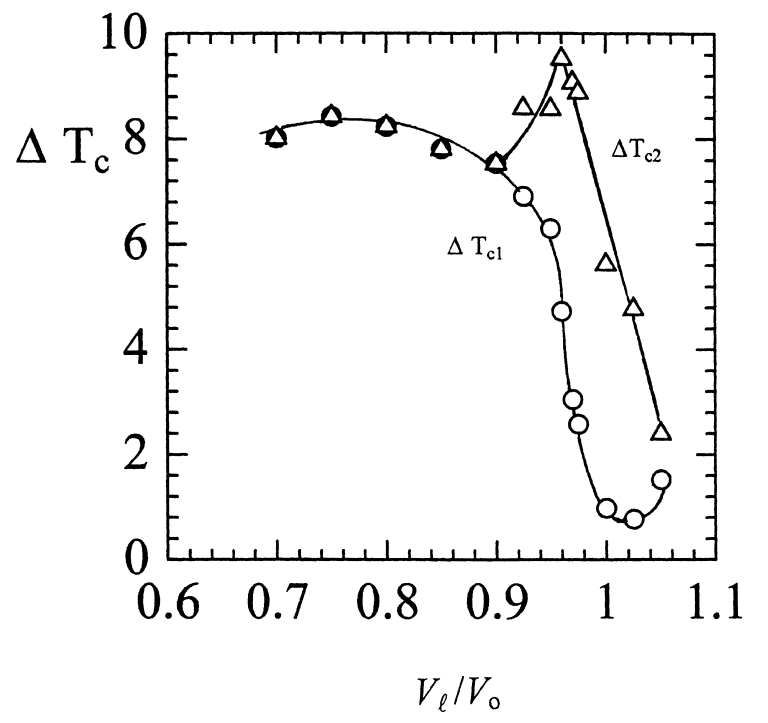

Fig. 9. The relation of the critical temperature difference and the volume ratio.

vection. In the case of the slender liquid bridges, the steady and axisymmetric convection transits directly to three-dimensional, oscillatory convection, and hence, $\Delta T_{\mathrm{c} 1}=\Delta T_{\mathrm{c} 2}$. These results are consistent with those obtained by the experiments. In the case of the fatter liquid bridge, there are two bifurcations, and $\Delta T_{\mathrm{c} 1} \neq \Delta T_{\mathrm{c} 2}$.

The dependence of critical applied temperature difference $\Delta T_{\mathrm{c}}$ on the liquid bridge volume is described by the second bifurcation $\Delta T_{\mathrm{c} 2}$ as usual. The feature of onset oscillation is thence divided into two branches separated by the peak, the slender and the fat liquid bridge branches, see for example, [3,9]. In the present case, The curve of second critical temperature difference $\Delta T_{\mathrm{c} 2}$ obtained in the calculation for the case of the oscillatory convection coincides qualitatively with the usual experimental results. Based on Fig. 9, the feature may be described by two branches, that is the slender and the fat liquid bridge branches separated by having only one bifurcation and two bifurcations. Then, the peak distribution is included in the fat bridge of liquid bridge.

\section{Discussions}

For checking the present numerical method, the results of thermocapillary convection in a cylindrical liquid bridge with $g=0$ and $l / d_{0}=10$ is compared with those obtained from linear stability analysis for infinite length, cylindrical liquid bridge and calculated by using three-dimensional, axisymmetric program. The results coincide quite well, except that there is $14 \%$ error at the meshes near the free surface. Another comparison is

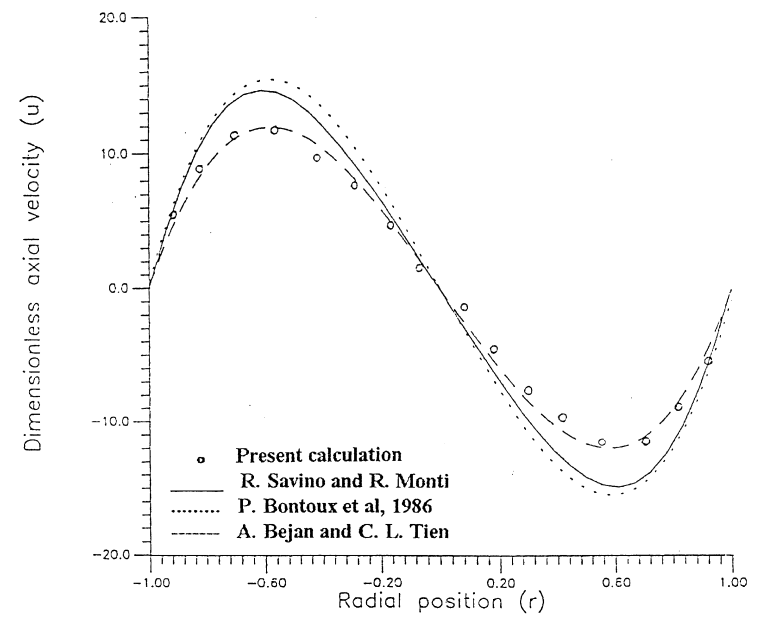

Fig. 10. The axial-velocity profile of the buoyancy convection in a azimuthal cylinder, $z / l=0.55$.

shown in Fig. 10. It shows the core velocity profile for buoyancy convection in a horizontal cylinder calculated by using the present program is consistent with the results of Bejan et al. [22] very well.

The onset of oscillatory thermocapillary convection in a fat liquid bridge of floating half zone of geometrical aspect ration $A=0.8$ and volume ratio $V_{1} / V_{0}=1.025$ has been analyzed for $10 \mathrm{cst}$ silicon oil of larger Prandtl number. There is only one bifurcation transition in the floating half zone convection for cases of the slender liquid bridge, but may have two bifurcation transitions for a fat liquid bridge. The first bifurcation transition is from the steady and axisymmetric convection to a steady and axial asymmetric convection, and then the second bifurcation transition relates to the transition from the steady and axial asymmetric to the oscillatory convection. These results show that, the rout of transition is from the steady and axi-symmetric convection via the steady and axial asymmetric convection to the oscillatory convection during the increasing of applied temperature difference in a fat liquid bridge. The coupling between temperature and velocity field is strong in this case because of the larger Prandtl number. The conclusion of the present paper does not agree with the hydrothermal instability, which relates with a traveling wave but not a steady state.

The studies of three-dimensional and time-dependent numerical simulation show that, the transition processes with two bifurcation appear in the critical region, which is defined by relatively smaller aspect ratio $A$ and relatively larger volume ratio $V_{1} / V_{0}$. A branch of fat liquid bridge with two bifurcation was obtained for a geometric aspect ratio $A=0.8$.

The conclusion of present paper implies that, the geometrical parameter $V_{1} / V_{0}$ is not only a sensitive critical parameter for onset of oscillatory thermocapillary 
convection, but is also important in studies of the mechanism which induces the different sort of bifurcation.

\section{Acknowledgements}

This research was started when Tang and Hu visited the Kyushu University via the exchange program between the Chinese Academy of Sciences and the Japanese Science Promotion Society and the invitation of Prof. Y. Sugioka, the President of the Kyushu University, respectively. They thank Prof. N. Imaishi for his kind cooperation during their visit. The research is partly supported by the National Natural Science Foundation of China (No. 19789201) and the project 95yu-34 of the Ministry of Science and Technology of China.

\section{References}

[1] Z.H. Cao, J.C. Xie, Z.M. Tang, W.R. Hu, The influence of buoyancy on the onset of oscillatory convection in a half floating zone, Adv. Space Res. 11 (7) (1991) 163.

[2] R. Monti, D. Castagnolo, P. Dell'aversana, G. Desiderio, S. Moreno, G. Evangelista, An experimental and numerical analysis of thermocapillary flow of silicon oils in a microfloating zone, 43rd Iaf-92-0918, 1992.

[3] W.R. Hu, J.Z. Shu, R. Zhou, Z.M. Tang, Influence of liquid bridge volume on the onset of oscillation in floating zone convection I. Experiment, J. Crystal Growth 142 (1994) 379.

[4] V.M. Shevtsova, J.C. Legros, Oscillatory convective motion in deformed liquid bridge, Phys. Fluids 10 (1998) 1621.

[5] Z.M. Tang, W.R. Hu, Influence of liquid bridge volume on the onset of oscillation in floating-zone convection III. Three-dimensional model, J. Crystal Growth 207 (1999) 239.

[6] Y.L. Yao, J.Z. Shu, J.C. Xie, W.R. Hu, A. Hirata, S.-I. Nishizawa, M. Sakurai, Transition of oscillatory floating half zone convection from Earth's gravity to microgravity, Int. J. Heat Mass Transfer 40 (1997) 2517.

[7] M. Sakurai, A. Hirata, Oscillatory thermocapillary convection in a liquid bridge under normal gravity and microgravity conditions - drop shaft experiments, Space Forum 3 (1998) 45.
[8] Q.S. Chen, W.R. Hu, V. Prasade, Effect of liquid bridge volume on the instability in small Prandtl-number half zones, J. Crystal Growth 203 (1999) 261.

[9] Q.S. Chen, W.R. Hu, Influence of liquid bridge volume on instability of floating half zone convection, Int. J. Heat Mass Transfer 42 (1998) 825.

[10] Ch.H. Chun, W. Wuest, Experiments on the transition from the steady to the oscillatory Marangoni-convection of a floating zone under reduced gravity effect, Acta Astronautica 6 (1979) 1073.

[11] D. Schwabe, A. Scharmann, Some evidence for the existence and magnitude of a critical Marangoni number for the onset of oscillatory flow in crystal growth melts, J. Crystal Growth 46 (1979) 125.

[12] G.P. Neiztel, K.T. Chang, D.F. Jankowski, H.D. Mittelmann, Linear-stability theory of thermocapillary convection in a model of the floating-zone crystal-growth process, Phys. Fluids A 5 (1993) 108.

[13] M. Wanschura, V.M. Shevtsova, H.C. Kuhlmann, H. Rath, Convective instability mechanism in thermocapillary liquid bridges, J. Phys. Fluids 7 (1995) 912.

[14] G. Chen, A. Lizee, B. Roux, Bifurcation analysis of the thermocapillary convection in cylindrical liquid bridge, J. Crystal Growth 180 (1997) 638.

[15] G.P. Neiztel, C.C. Law, D.F. Jankowski, H.D. Mittelmann, Energy stability of thermocapillary convection in a model of the floating zone crystal-growth process. II: nonaxisymmetric disturbance, Phys. Fluid A 3 (1991) 2841.

[16] R. Savino, R. Monti, Oscillatory Marangoni convection in cylindrical liquid bridgs, Phys. Fluid 8 (1996) 2906.

[17] S. Yasuhiro, T. Sato, N. Imaishi, Three dimensional oscillatory Marangoni flow in half-zone of $P r=1.02$ fluid, Microgravity Sci. Technol. 10 (1997) 144.

[18] M.K. Smith, S.H. Davis, Instabilities of dynamic thermocapillary liquid layers. Part 1. Convective instability, J. Fluid Mech. 132 (1983) 119.

[19] M. Levenstam, G. Amberg, Hydrodynamical instabilities of thermocapillary flow in a half-zone, J. Fluid Mech. 297 (1995) 357.

[20] Z.M. Tang, W.R. Hu, The onset process of thermocapillary oscillatory convection in a half floating zone, IAF-98J.4.04, 49th IAF Congress.

[21] Q.S. Chen, W.R. Hu, Instability from steady and axisymmetric to steady and asymmetric floating half zone convection in a fat liquid bridge of large Prandtl number, Chinese Phys. Lett. 16 (11) (1999) 822.

[22] A. Bejan, C.L. Tien, Laminar natural convection heat transfer in a horizontal cavity with different end temperature, Int. J. Heat Mass Transfer 23 (1980) 1127. 